See discussions, stats, and author profiles for this publication at: https://www.researchgate.net/publication/326840468

Simultaneous determination of thermal conductivity and shear viscosity using two-gradient non-equilibrium molecular dynamics simulations

Article in Molecular Physics · August 2018

DOl: 10.1080/00268976.2018.1504134

CITATIONS

3 authors, including:

Martin Lautenschlaeger

Technische Universität Kaiserslautern

4 PUBLICATIONS 2 CITATIONS

SEE PROFILE

Some of the authors of this publication are also working on these related projects:

Computational Molecular Engineering View project
READS

55

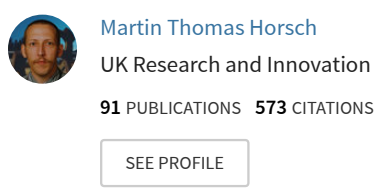




\title{
PRE-PRINT
}

\section{Simultaneous Determination of Thermal Conductivity and Shear Viscosity Using Two-Gradient Non-Equilibrium Molecular Dynamics Simulations}

\author{
Martin P. Lautenschlaeger ${ }^{\mathrm{a}}$, Martin Horsch ${ }^{\mathrm{a}}$ and Hans Hasse ${ }^{\mathrm{a}}$ \\ ${ }^{a}$ Laboratory of Engineering Thermodynamics (LTD), University of Kaiserslautern, \\ Erwin-Schrödinger-Straße 44, 67663 Kaiserslautern, Germany
}

\author{
ARTICLE HISTORY \\ Compiled August 8, 2018
}

\begin{abstract}
A method for the simultaneous determination of the thermal conductivity $\lambda$ and the shear viscosity $\eta$ of fluids by non-equilibrium molecular dynamics simulations is presented and tested using the Lennard-Jones truncated and shifted fluid as example. The fluid is studied under the simultaneous influence of a temperature gradient $\partial T / \partial y$ and a velocity gradient $\partial \boldsymbol{v} / \partial y$ and the resulting heat flux and momentum flux are measured to determine $\lambda$ and $\eta$. The influence of the magnitude of $\partial T / \partial y$ and $\partial \boldsymbol{v} / \partial y$ on $\lambda$ and $\eta$ is investigated. The cross-effects are negligible, even for large gradients. The same holds for the influence of $\partial T / \partial y$ on $\lambda$. However, there is a significant influence of $\partial \boldsymbol{v} / \partial y$ on $\eta$, i.e. shear-thinning. The two-gradient method is applied in different ways: for small $\partial T / \partial y$ temperature-averaged values of $\lambda$ and $\eta$ are obtained. As $\partial T / \partial y$ has no significant influence on the results, simulations with large $\partial T / \partial y$ are evaluated using the local-equilibrium assumption, such that values are obtained at different temperatures in a single simulation. In addition to the results for $\lambda$ and $\eta$, also results for the self-diffusion coefficient $D$ are determined from evaluating the mean squared displacement. The new two-gradient method is robust, efficient, and yields accurate results.
\end{abstract}

\section{KEYWORDS}

NEMD; thermal conductivity; shear viscosity; self-diffusion coefficient;

Lennard-Jones; LJTS; simple fluid; shear flow; shear-thinning; cross-effects

\section{Introduction}

Molecular dynamics (MD) simulations are widely used for determining transport properties of fluids. These simulations are still computationally expensive. Thus, in the present work a new efficient method for determining transport properties of fluids with MD simulations was developed and tested.

Transport properties can be determined with equilibrium molecular dynamics (EMD) or with non-equilibrium molecular dynamics (NEMD) simulations. In EMD usually the Green-Kubo method is used. For NEMD different techniques have been developed, where either a temperature gradient [4 7] or a velocity gradient [8 14] is

The Version of Record of this manuscript has been published and is available in MOLECULAR PHYSICS 06 Aug 2018 http://www.tandfonline.com/doi/full/10.1080/00268976.2018.1504134

CONTACT Martin P. Lautenschlaeger. Email: Martin.Lautenschlaeger@mv.uni-kl.de 
prescribed and the corresponding flux is calculated, or vice versa. The latter is sometimes called reverse NEMD [15, 16]. For a general introduction into the determination of transport properties by MD simulations and a more comprehensive overview, see [17-19].

The main new feature of the method presented here is that a temperature gradient $\partial T / \partial y$ and a velocity gradient $\partial \boldsymbol{v} / \partial y$ are applied simultaneously such that the thermal conductivity $\lambda$ and the shear viscosity $\eta$ can be determined in a single simulation run. The new method is therefore referred to as 'two-gradient method' in the following.

It is well known from transport theory that a superposition of gradients may lead to cross-effects. I.e. the heat flux $\boldsymbol{j}$ is not independent of the velocity gradient $\partial \boldsymbol{v} / \partial y$ and the momentum flux $\boldsymbol{\sigma}$ is not independent of the temperature gradient $\partial T / \partial y$. The twogradient method can be used to study such cross-effects as well as the dependency of $\lambda$ on $\partial T / \partial y$ and that of $\eta$ on $\partial \boldsymbol{v} / \partial y$. It is tested here for the Lennard-Jones truncated and shifted (LJTS) fluid. Even for large gradients, the cross-effects are found to have no significant influence. The same holds for the influence of $\partial T / \partial y$ on $\lambda$. In contrast, the well-known shear-thinning, i.e. a reduction of $\eta$ with increasing $\partial \boldsymbol{v} / \partial y$ is observed.

As an additional feature, the self-diffusion coefficient $D$ is determined simultaneously with $\lambda$ and $\eta$ from the mean squared displacement.

The two-gradient method is applied here in different ways. They have in common that periodic boundary conditions are applied such that the simulation volume is at constant pressure.

1) Both gradients are small and the results for $\lambda, \eta$, and $D$ are averaged along the gradients and assigned to a single state. This is called 'global method' here.

2) The temperature gradient is large and the velocity gradient is small. The assumption of local equilibrium [4, 20] is applied and results for $\lambda, \eta$, and $D$ are obtained for different temperatures in a single simulation run. This is called 'local method' here and it is based on the ideas proposed in [22 24].

In both the global and the local method, the gradient is applied by the specification of states in spatially separated control volumes. The fluid between these control volumes is unconstrained. This may lead to viscous heating for large values of the velocity gradient.

3) Therefore, for systematic studies of the influence of the velocity gradient a variant of the global method is used in which a small and perfectly linear temperature gradient is applied via a series of thermostats. Thus, the effects of viscous heating are prevented and the velocity gradient can be increased up to large values. This is called 'shear rate study method' here.

The applicability of all methods is tested by calculating $\lambda, \eta$, and $D$ of the LJTS fluid. However, it is not in the scope of the present work to perform a comprehensive study of transport properties of the LJTS fluid. The global method is tested for one subcritical and one supercritical isotherm. The local method is tested for a series of supercritical states along a single isobar. The shear rate study method is tested at a single liquid state point at low temperature. Good agreement with literature data is observed in all cases.

The two-gradient method is simple, intuitive, and easy to implement. Nonetheless, it is robust, efficient, accurate, and flexible. It is described here for pure simple fluids. But with only minor adaptions it can also be applied to more complex fluids and fluid mixtures.

This paper is organized as follows: in Section 2 the two-gradient method is introduced and the three variants mentioned above are discussed. In Section 3 the simulation setup that was used in the present work and the LJTS force field are specified. In Section 
4 preliminary investigations on the choice of the two gradients and their influence on the transport properties are described. Results of tests of all three variants of the two-gradient method are presented in Section 5. In that section also the results of the studies on shear-thinning are reported. Conclusions are drawn in Section 6.

\section{Two-Gradient Method}

\subsection{Overview}

In the two-gradient method a simulation box with periodic boundary conditions in all directions is used. It is completely filled with fluid. The mean particle density in the simulation box is prescribed. There are no walls, so that surface effects are excluded. Due to the periodic boundary conditions, the pressure in the box fluctuates around a constant value. The magnitude of the pressure depends on the choice of the initial density and the gradients that are applied.

Figure 1 shows a sketch of the setup. It is symmetrical in $y$-direction. The shear flow is in $+x$-direction. The temperature and the velocity are prescribed in certain control volumes using Gaussian isokinetic thermostats [25] and constraining the equations of motion, respectively. The size of the control volumes for the temperature and the velocity may differ.

The following summary description holds for the global and the local method. For the shear rate study method some modifications are applied that are described further below. The gradients are applied as follows: in the control volumes at the two borders of the box in $y$-direction, the mean temperature is high ( $\left.T_{\text {hot }}\right)$ and the mean velocity is zero $\left(\boldsymbol{v}_{x}=0\right)$. In the control volumes in the middle of the box the mean temperature is low $\left(T_{\text {cold }}\right)$ and the mean velocity is high $\left(\boldsymbol{v}_{x}=v_{\max }\right)$. No constraints other than those are applied. The temperature and velocity profiles outside the control volumes are a response. For determining the profiles, the simulation box is uniformly discretized in $y$-direction so that flat bins with equal thickness are obtained. In each bin the scalar properties number density $\rho$ and temperature $T$, the vectors velocity $\boldsymbol{v}$ and heat flux $\boldsymbol{j}$, as well as the stress tensor $\boldsymbol{\sigma}$ are calculated separately as block averages. From those $\lambda$ and $\eta$ are determined in the unconstrained regions using transport ansatzes, i.e. Fourier's and Newton's law, respectively. Details are given below for the case of pure simple fluids.

\subsection{Fundamentals}

Directed velocities. The instantaneous velocity $\boldsymbol{v}_{i}$ of each particle $i$ can be written as

$$
\boldsymbol{v}_{i}=\boldsymbol{v}_{\mathrm{dir}}+\boldsymbol{v}_{i, \text { therm }}
$$

The first term on the right-hand side of Equation 1 is the directed macroscopic flow velocity. The second term is the undirected thermal velocity. It is determined as the time-average of the instantaneous velocities. Details are given in Appendix B

Temperature. The temperature is calculated from the kinetic energy of the undirected 

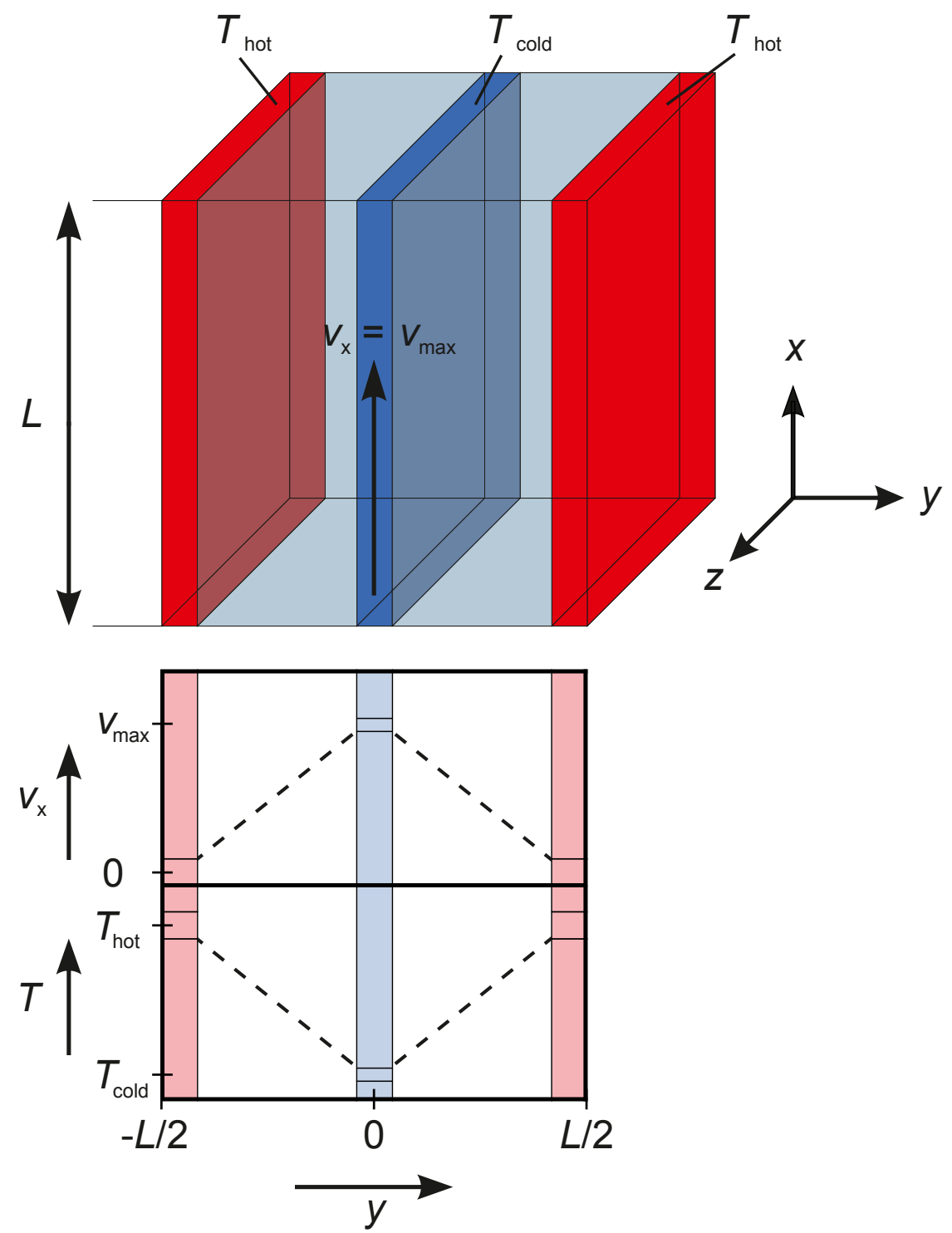

Figure 1.: Scheme of the simulation setup used for the two-gradient method. The control volumes in which the temperature and the velocity are prescribed are marked in blue and red. The shear flow is in $+x$-direction. Exemplary temperature and velocity profiles are depicted at the bottom. The dashed lines indicate responses. Within the control volumes the mean temperature and the mean velocity are adjusted leading to an upper and lower bound value indicated by the solid vertical lines. 
thermal velocity:

$$
T=\frac{\sum_{i}^{N_{\text {bin }}} m_{i} \boldsymbol{v}_{i, \text { therm }}^{2}}{3 N_{\text {bin }} k_{\mathrm{B}}}
$$

where $N_{\text {bin }}$ is the number of particles per bin, $m_{i}$ is the mass of a single particle, and $k_{\mathrm{B}}$ is the Boltzmann's constant.

Heat flux vector and stress tensor. The local heat flux vector $\boldsymbol{j}$ is calculated following the approach by Irving and Kirkwood [26]:

$$
\begin{aligned}
& \boldsymbol{j}_{k}=\frac{1}{V_{\text {bin }}}\left[\sum_{i}^{N_{\text {bin }}}\left(\frac{1}{2} m_{i} \boldsymbol{v}_{i, l, \text { therm }}^{2}\right) \boldsymbol{v}_{i, k, \text { therm }}+\frac{1}{2} \sum_{i}^{N_{\text {bin }}} \sum_{j \neq i}^{N_{\text {Neighbor }}} \boldsymbol{r}_{i j, k}\left(\boldsymbol{F}_{i j, l} \boldsymbol{v}_{i, l, \text { therm }}\right)\right. \\
& \left.+\frac{1}{2} \sum_{i}^{N_{\mathrm{bin}}} \sum_{j \neq i}^{N_{\text {Neighbor }}} \Phi\left(r_{i j}\right) \boldsymbol{v}_{i, k, \text { therm }}\right] \text {. }
\end{aligned}
$$

$\boldsymbol{j}_{k}$ is the component of the heat flux vector in $k$-direction $(k=x, y, z) . V_{\mathrm{bin}}$ is the bin volume. $N_{\text {Neighbor }}$ is the number of interacting neighbors per particle $i . \boldsymbol{v}_{i, k}$ is the instantaneous velocity of particle $i$ in $k$-direction. $\boldsymbol{r}_{i j, k}$ and $\boldsymbol{F}_{i j, l}$ are the $k$ - and $l$-component of the distance and the force vector between the two particles $i$ and $j$, respectively. $\Phi\left(r_{i j}\right)$ is the interaction potential between the particles $i$ and $j$.

The local stress tensor $\boldsymbol{\sigma}$ is also calculated following the approach by Irving and Kirkwood [26]:

$$
\boldsymbol{\sigma}_{k l}=\frac{1}{V_{\mathrm{bin}}}\left[\sum_{i}^{N_{\text {bin }}} m_{i} \boldsymbol{v}_{i, k, \text { therm }} \boldsymbol{v}_{i, l, \text { therm }}+\frac{1}{2} \sum_{i}^{N_{\text {bin }}} \sum_{j \neq i}^{N_{\text {Neighbor }}} \boldsymbol{r}_{i j, k} \boldsymbol{F}_{i j, l}\right] .
$$

The index $k l$ is the component of the stress tensor in the $k l$-plane $(k, l=x, y, z)$. The hydrodynamic pressure follows as

$$
p=\frac{1}{3} \operatorname{tr}(\boldsymbol{\sigma}) .
$$

Post-processing analysis. Profiles of the observables number density $\rho$, velocity $\boldsymbol{v}_{x}$, temperature $T$, pressure $p$, shear stress $\boldsymbol{\sigma}_{x y}$, and heat flux $\boldsymbol{j}_{y}$ are determined as discretized functions of the coordinate $y$. They are time-averaged within the production run. An example is presented in Figure 2, where the symmetry in the $y$-direction is indicated with vertical black lines, i.e. the axis origin. The control volumes are depicted as red- and blue-shaded regions. These are excluded from the analysis, since the curvature of the profiles is directly affected by the thermostats and the velocity control there.

The left $(y<0)$ and the right $(y \geq 0)$ half of the system are firstly treated independently of each other. Least squares regressions are conducted in the unconstrained regions separately on the left and the right side. Second-order polynomials are fitted to the temperature and velocity profiles, while first-order polynomials are fitted to the heat flux and the shear stress profiles. $\lambda$ and $\eta$ are determined bin-wise from Fourier's 

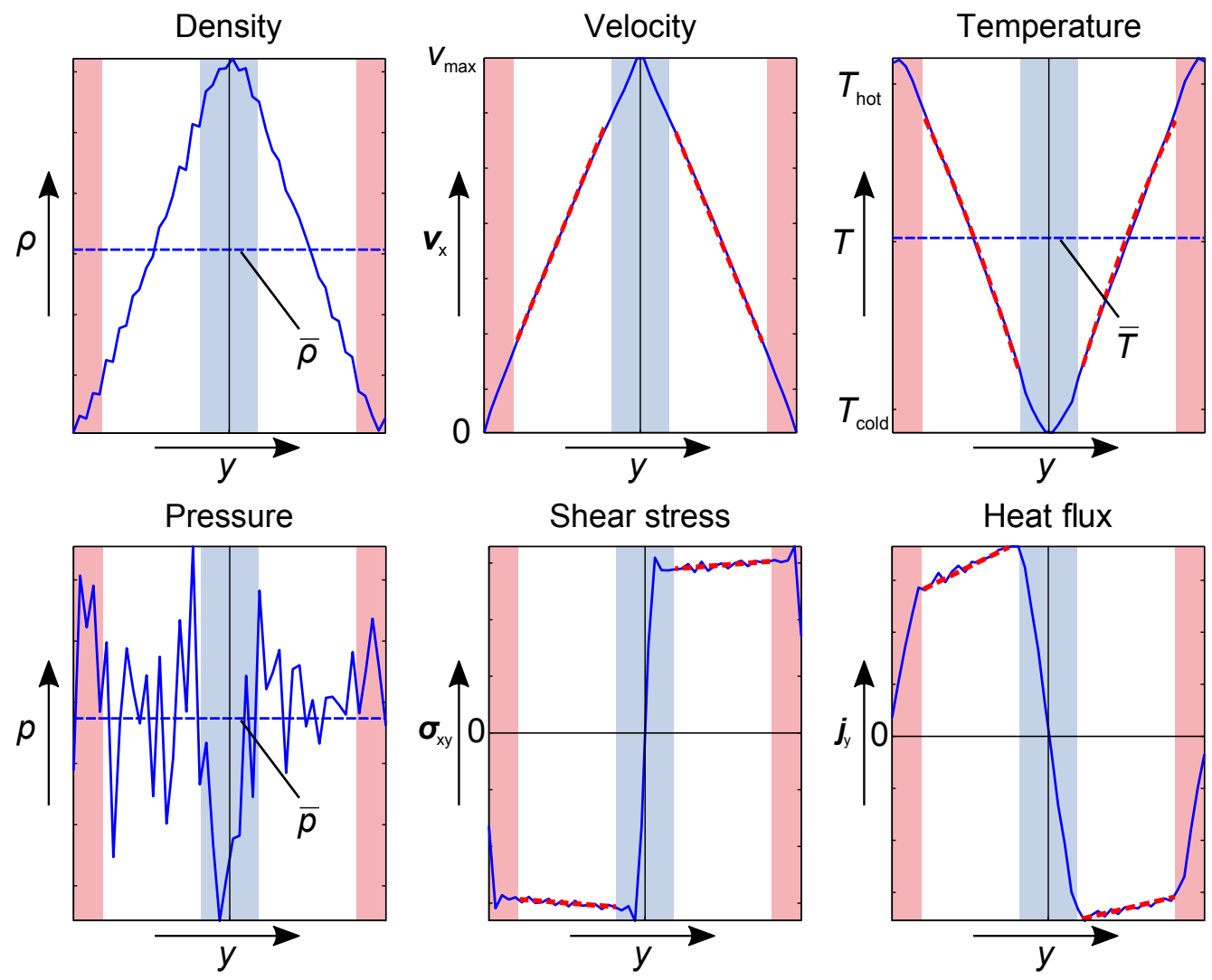

Figure 2.: Overview over the primary observables that are measured in simulations with the two-gradient method. The bin-wise results are depicted with the blue solid lines. The mean values of the density $\bar{\rho}$, the temperature $\bar{T}$, and the pressure $\bar{p}$ are indicated with the vertical blue dashed lines. The regression fits are shown with the red dashed lines. They are just conducted in the unconstrained regions between the control volumes, which are indicated by the shaded areas. 
law

$$
\boldsymbol{j}_{y}=-\lambda \frac{\partial T}{\partial y},
$$

and Newton's law

$$
\boldsymbol{\sigma}_{x y}=-\eta \frac{\partial \boldsymbol{v}_{x}}{\partial y}=-\eta \dot{\gamma},
$$

respectively, using the values obtained from the least squares regressions.

The self-diffusion coefficient vector $\boldsymbol{D}_{k}$ is determined bin-wise in the unconstrained regions. It is calculated from the mean squared displacement. The calculation accounts for the convective flow; cf. Equation 8 .

$$
\boldsymbol{D}_{k}=\frac{1}{2 N_{\mathrm{bin}} \Delta t}\left[\sum_{i}^{N_{\text {bin }}}\left(\boldsymbol{r}_{i, k}(t+\Delta t)-\boldsymbol{r}_{i, k}(t)-\left\langle\boldsymbol{v}_{k, \operatorname{dir}}\right\rangle \Delta t\right)^{2}\right] .
$$

$\boldsymbol{r}_{i, k}$ is the $k$-component of the coordinate of particle $i$. Here, for each particle the initial position is compared with the position after the time period $\Delta t$. The last term on the right-hand side takes the convective flow into account. The angle brackets denote the time-average of the directed velocity in the $k$-direction.

In accordance with the literature, the isotropic self-diffusion coefficient $D$ is defined as:

$$
D=\frac{1}{3}\left(\boldsymbol{D}_{x}+\boldsymbol{D}_{y}+\boldsymbol{D}_{z}\right) .
$$

\subsection{Variants of the Two-Gradient Method}

The two-gradient method can be applied in three different ways. They differ in terms of accuracy and efficiency. Moreover, different temperature and velocity gradients are applied.

Global method. The temperature gradient and the velocity gradient are small. $\lambda, \eta$, and $D$ are calculated in each bin and averaged along the gradients in the unconstrained regions. The averages are assigned to a single thermodynamic state point such as $\lambda(\bar{T}, \bar{\rho}), \eta(\bar{T}, \bar{\rho})$, and $D(\bar{T}, \bar{\rho})$. The bar represents the spatial average; cf. Figure 2 . The accuracy of the global method is high.

Local method. The temperature gradient is large and the velocity gradient is small. Thereby, a series of temperature-dependent states is created along the $y$-direction. The assumption of local equilibrium [4, 20] is applied. $\lambda, \eta$, and $D$ are calculated in each bin. A mean value is determined from the two corresponding opposed symmetric bins on the left and the right side. Accordingly, the transport properties are assigned to the mean thermodynamic state of the two corresponding symmetric bins. Within a single simulation run a series of transport properties is determined. Thus, the efficiency increases by one order of magnitude compared to the global method; cf. [22, 24]. The accuracy is only slightly lower than that of the global method.

For both aforementioned variants the temperature and the velocity are only prescribed in the control volumes at the borders and in the middle of the simulation box. 
The fluid inbetween is unconstrained. This may lead to viscous heating for large values of the velocity gradient and is avoided in the method that is described in the following. Shear rate study method. A small and perfectly linear temperature gradient is applied via a series of Gaussian isokinetic thermostats. Thus, the effects of viscous heating are prevented and the velocity gradient $\dot{\gamma}$ can be increased up to large values. Thereby, shear effects on different physical properties can be studied. The post-processing analysis is equal to that of the global method. The transport properties are determined as $\lambda(\bar{T}, \bar{\rho}, \dot{\gamma}), \eta(\bar{T}, \bar{\rho}, \dot{\gamma})$, and $D(\bar{T}, \bar{\rho}, \dot{\gamma})$. The accuracy is comparable to the global method, except for $\lambda$, where strong perturbations by the large number of thermostats lead to instabilities of the heat flux, and thus a large scattering, especially at high shear rates.

\section{Simulation Setup and Model}

Simulation setup. In the present study, the simulation box is cubic. The edge length $L$ is chosen so that the box contains about 10,000 particles. This leads to larger systems for low densities. To avoid finite size effects the minimum edge length is set to $L_{\mathrm{min}}=$ $24 \sigma[19,27,28]$.

The box is discretized with 48 bins. The corresponding bin thickness is $\Delta y=L / 48$, resulting in the minimum bin thickness $\Delta y_{\min }=0.5 \sigma$.

A simulation run consists of initially 500,000 time steps in which a steady state is established, i.e. a stable temperature and velocity gradient are built up. Subsequently, a production run of 2,000,000 time steps is conducted. Block-averaging is applied over 10,000 time steps each (cf. [17]). The time step is $\Delta \tau=0.002 \sigma \sqrt{M / \varepsilon}$.

The simulations are carried out with a version of the MD code ls1 mardyn [29] that was substantially extended in the present work.

Molecular model. The two-gradient method is tested with the LJTS fluid. It is based on the Lennard-Jones (LJ) potential $u^{\mathrm{LJ}}$ that is the most frequently applied model in molecular simulation history. Both models are known to reproduce thermo-physical properties of simple nonpolar fluids accurately [17, 30 32].

The LJTS potential is

$$
u^{\mathrm{LJTS}}\left(r_{i j}\right)=\left\{\begin{array}{ll}
u^{\mathrm{LJ}}\left(r_{i j}\right)-u^{\mathrm{LJ}}\left(r_{\mathrm{c}}\right), & r_{i j}<r_{\mathrm{c}} \\
0, & r_{i j} \geq r_{\mathrm{c}}
\end{array},\right.
$$

where

$$
u^{\mathrm{LJ}}\left(r_{i j}\right)=4 \varepsilon\left[\left(\sigma / r_{i j}\right)^{12}-\left(\sigma / r_{i j}\right)^{6}\right] .
$$

$\varepsilon$ is the energy parameter, describing dispersive attraction and $\sigma$ is the size parameter describing the repulsion. $r_{i j}$ is the distance between the two LJTS particles $i$ and $j . r_{\mathrm{c}}$ is the cut-off radius, which is the maximal distance for which the attraction is considered. It is $2.5 \sigma$ throughout the present work. In the following all observables are given in reduced units, cf. Table 1 .

\section{Preliminary Investigation}

The influence of the temperature and velocity gradient on the accuracy of the twogradient method is studied. Therefore, one gradient is varied while the other gradient 
Table 1.: Definition of the physical quantities in reduced units (marked by the asterisk). The corresponding observable carrying a dimension is plain. $M$ is the mass of a single particle.

\begin{tabular}{|c|c|c|c|}
\hline Length & $x^{*}=\frac{x}{\sigma}$ & Velocity & $v^{*}=\frac{v}{\sqrt{\varepsilon / M}}$ \\
\hline Time & $\tau^{*}=\frac{\tau}{\sigma \sqrt{M / \varepsilon}}$ & Shear rate & $\gamma^{*}=\frac{\gamma}{\sqrt{\varepsilon / M} / \sigma}$ \\
\hline Mass & $m^{*}=\frac{m}{M}$ & Energy flux & $\boldsymbol{j}^{*}=\frac{j}{\varepsilon \sqrt{\varepsilon / M} / \sigma^{3}}$ \\
\hline Density & $\rho^{*}=\rho \sigma^{3}$ & Thermal conductivity & $\lambda^{*}=\frac{\lambda}{k_{\mathrm{B}} \sqrt{\varepsilon / M} / \sigma^{2}}$ \\
\hline Temperature & $T^{*}=\frac{T}{\varepsilon / k_{\mathrm{B}}}$ & Shear viscosity & $\eta^{*}=\frac{\eta}{\sqrt{\varepsilon M} / \sigma^{2}}$ \\
\hline Stresses & $\boldsymbol{\sigma}^{*}=\frac{\boldsymbol{\sigma}}{\varepsilon / \sigma^{3}}$ & Self-diffusion coeff. & $D^{*}=\frac{D}{\sqrt{\varepsilon / M} / \sigma}$ \\
\hline
\end{tabular}

is fixed. Additionally, cross-effects are investigated.

The preliminary investigations are conducted at a subcritical $\left(\bar{T}^{*}=1.0, \bar{\rho}^{*}=0.8\right)$ and a supercritical $\left(\bar{T}^{*}=2.0, \bar{\rho}^{*}=0.8\right)$ thermodynamic state.

The influence of the temperature gradient is shown in Figure 3 . It is studied in the range of $\partial T^{*} / \partial y^{*}=[0.005 ; 0.1]$. The shear rate is fixed to $\dot{\gamma}^{*}=0.03$. The results are shown for stable state densities only. Thus, for $\bar{T}^{*}=1.0$ and $\partial T^{*} / \partial y^{*}=0.1$ no data was calculated. There is hardly any correlation between $\partial T^{*} / \partial y^{*}$ and the transport properties. Thus, no remarkable cross-effect regarding the momentum flux can be observed. However, for $\partial T^{*} / \partial y^{*} \lesssim 0.01$ the estimated uncertainty of $\lambda^{*}$ is comparably large which is shown by the error bars. In the following, the temperature gradient is set to $\partial T^{*} / \partial y^{*} \geq 0.025$.

The influence of the velocity gradient is shown in Figure 4. It is studied in the range of $\dot{\gamma}^{*}=[0.01 ; 1.0]$. The temperature gradient is fixed to $\partial T^{*} / \partial y^{*}=0.03$. The influence of $\dot{\gamma}^{*}$ on $\eta^{*}$, i.e. the shear-thinning, and $D^{*}$ is obvious. However, no remarkable cross-effect regarding the heat flux is observed. The effect on $D^{*}$ is not considered as a cross-effect here, since it is not calculated with a transport ansatz; cf. Eqs. (8) \& (9). For $\dot{\gamma}^{*} \lesssim 0.1$ plateau values, i.e. the corresponding equilibrium values for $\eta^{*}$ and $D^{*}$ are found. In the following, the velocity gradient is set to $\dot{\gamma}^{*}=0.03$ which is a good compromise between the uncertainties and the quasi-equilibrium state.

The basic effects that were described above and can be found in Figure 3 and Figure 4 have also been reported in the literature [33, 35]: 1) $\lambda$ is independent of the temperature gradient within the statistical uncertainties [6, 7]. 2) $\eta$ decreases with increasing shear rate. This is associated with a structural change from an amorphous liquid to a stringlike pattern which results in a reduction of the shear stress. 3) The shear-thinning is more pronounced for low temperatures. 4) $D$ behaves inversely proportional to $\eta$, which can be explained with the Stokes-Einstein relation. Thus, at low temperatures $D$ increases with increasing shear rate.

The preliminary investigations provide a first indication for the influence of the temperature and velocity gradients on the analysis of the transport properties. The cross-effects are found to have no significant influence for the gradients studied here. This underpins the applicability of the two-gradient method for the simultaneous calculation of $\lambda, \eta$, and $D$. 


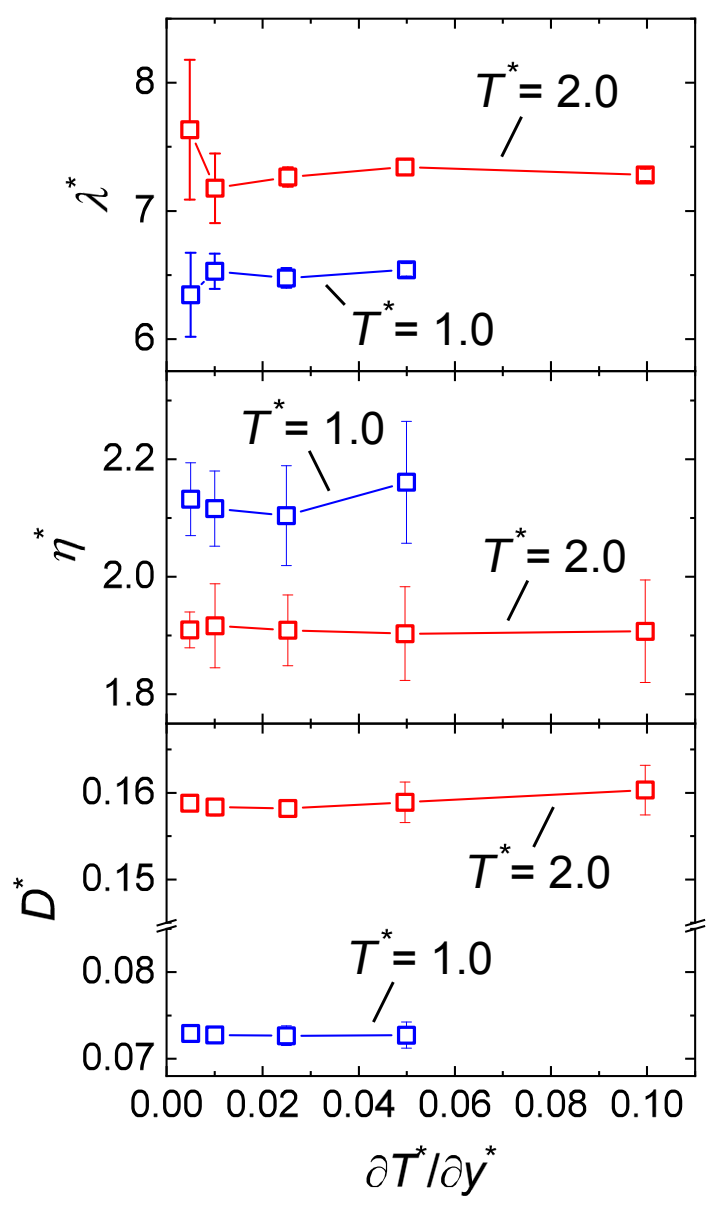

Figure 3.: Influence of the temperature gradient on the transport properties. The results are calculated for the density $\bar{\rho}^{*}=0.8$ along the two isotherms $\bar{T}^{*}=1.0$ (blue line) and $\bar{T}^{*}=2.0$ (red line). The estimated uncertainties of our results are depicted by the error bars. 


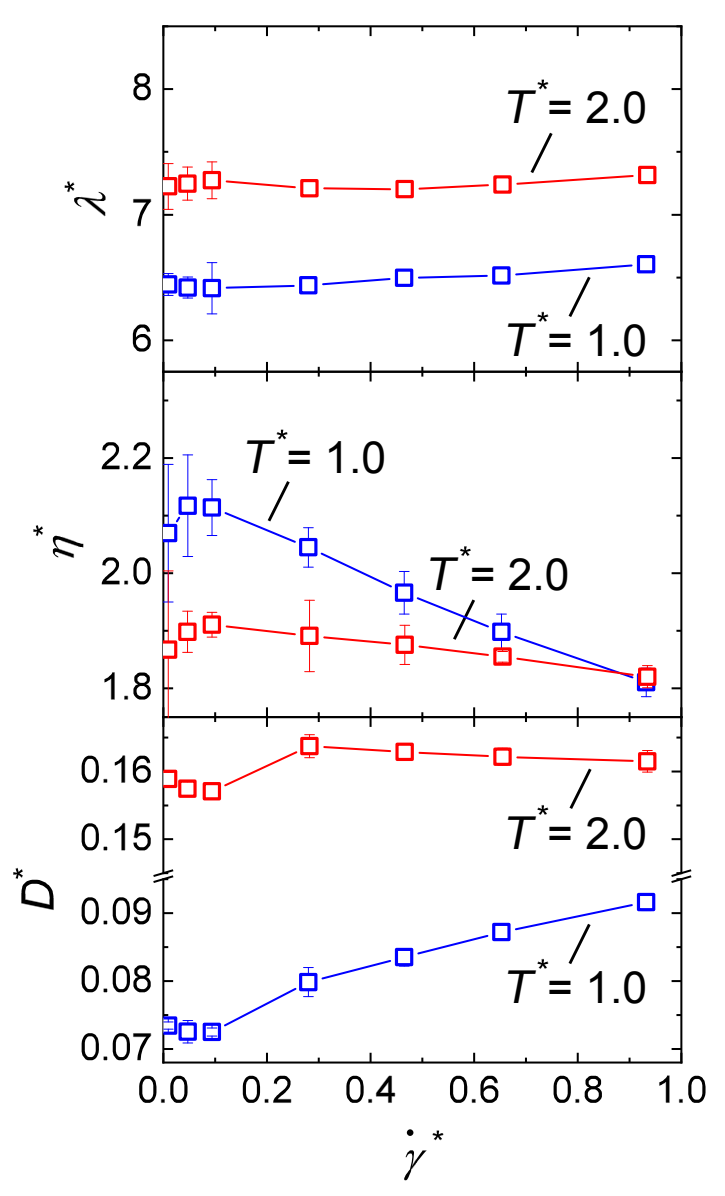

Figure 4.: Influence of the velocity gradient on the transport properties. The results are calculated for the density $\bar{\rho}^{*}=0.8$ along the two isotherms $\bar{T}^{*}=1.0$ (blue line) and $\bar{T}^{*}=2.0$ (red line). The estimated uncertainties of our results are depicted by the error bars. 


\section{Main Investigation}

The aforementioned variants of the two-gradient method are tested and evaluated here. The results are compared to literature data. Since there is hardly any data for the LJTS in the literature, data for the LJ fluid is used. This is in accordance with several authors [27, 36, 41] that have shown that for $r_{\mathrm{c}}^{*} \geq 2.5$ a truncation of the LJ potential has no effect on the transport properties.

\subsection{Global Method}

The temperature gradient and the velocity gradient are set to $\partial T^{*} / \partial y^{*}=0.03$ and $\dot{\gamma}^{*}=0.03$, respectively. $\lambda, \eta$, and $D$ are calculated along the subcritical isotherm $\bar{T}^{*}=1.0$ and the supercritical isotherm $\bar{T}^{*}=2.0$ over a wide range of densities $\bar{\rho}^{*}=[0.02 ; 1.1]$. No data is calculated in the two-phase region, i.e. $\bar{\rho}^{*} \approx[0.1 ; 0.55]$ for $\bar{T}^{*}=1.0$.

In Figure 5 (top) the values for $\lambda, \eta$, and $D$ as well as the literature data are depicted. The color indicates the temperature: $\bar{T}^{*}=1.0$ (blue), $\bar{T}^{*}=2.0$ (red). In Figure 5 (bottom) the relative deviations between correlations of our data and the literature data are shown. The color coding and the symbols have the same meaning as above. The dashed reference line represents the correlation of our data.

The statistical uncertainties of $\lambda, \eta$, and $D$ are $1 \%, 3 \%$, and $0.5 \%$, respectively, for the two isotherms shown here. They are equal or even slightly lower than the respective uncertainties of the broadly acknowledged literature data that was used for the comparison.

The results agree very well over a wide range of densities. For dense fluids with $\bar{\rho}^{*}>0.2$ the maximum deviation is below $10 \%$. This corresponds to the common data scattering in the broad literature. The largest deviations are observed for low densities, i.e. $\bar{\rho}^{*} \leq 0.2$. Here, the absolute values for $\lambda$ and $\eta$ are small. Thus, small absolute deviations lead to large relative deviations. The same is found for $D$ at higher densities. Nevertheless, the results indicate that the two-gradient method is not well suited for studying states with low densities, cf. hatched area in Figure 5. For these states, the directed macroscopic flow velocity and the thermal velocity are hard to differentiate due to poor statistics. This has a direct consequence on the calculation of the temperature, the heat flux, and the stress profiles and thus on $\lambda$ and $\eta$.

\subsection{Local Method}

The temperature gradient and the velocity gradient are set to $\partial T^{*} / \partial y^{*}=0.1$ and $\dot{\gamma}^{*}=0.03$, respectively. The mean temperature is $\bar{T}^{*}=2.0$ and the mean density is $\bar{\rho}^{*}=0.8 . \lambda, \eta$, and $D$ are calculated locally for a series of different thermodynamic states $\left(T^{*} \in[1.5 ; 2.5], \rho^{*} \in[0.75 ; 0.85]\right)$.

First of all, the criterion of the weak local equilibrium is verified [20]. I.e. it is ensured that the local pressure along the temperature gradient is equal to the corresponding equilibrium pressure. This is shown in the deviation plot presented in Figure 6. The dashed reference line represents the equilibrium pressure predicted from the equation of state (EOS) by Thol et al. [45. The estimated uncertainty of the local pressure calculation is $1 \%$ and indicated by the error bars. It is shown, that the local pressures agree perfectly with the predictions. The deviation is $0.2 \%$ at its maximum. As indicated by several authors [7, 22, 24, this is a strong indicator for the local transport 

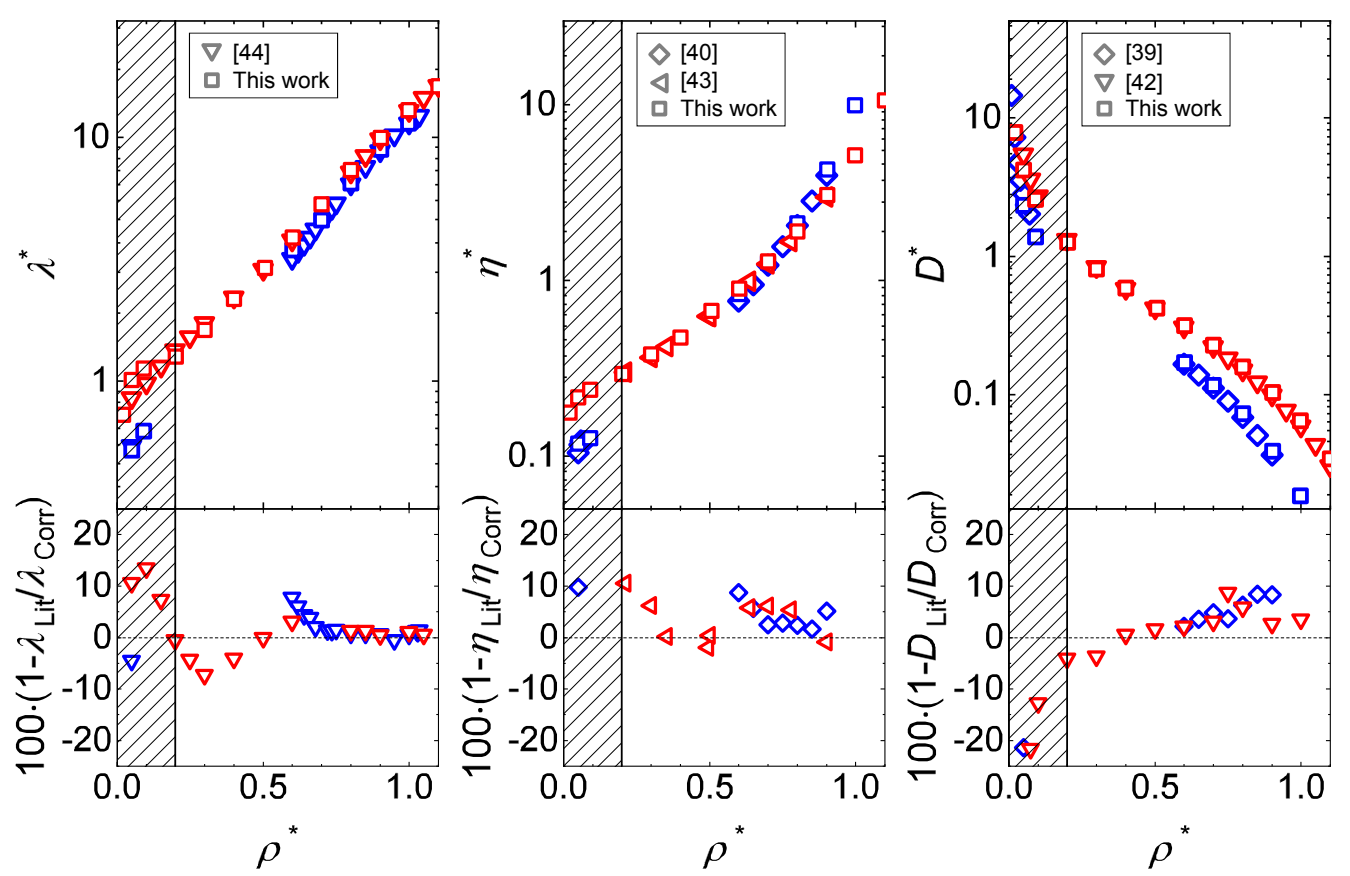

Figure 5.: Comparison of the transport properties determined using the global method with literature data over a wide range of densities. The plots at the top show absolute values, while at the bottom the relative deviations of the literature data from a correlation based on our results are shown. The blue symbols represent values along the isotherm $\bar{T}^{*}=1.0$, while the red symbols represent values along the isotherm $\bar{T}^{*}=2.0$. The symbols are listed in the respective legends. As indicated by the hatched area the two-gradient method should not be applied for low densities $\bar{\rho}^{*} \leq 0.2$. 


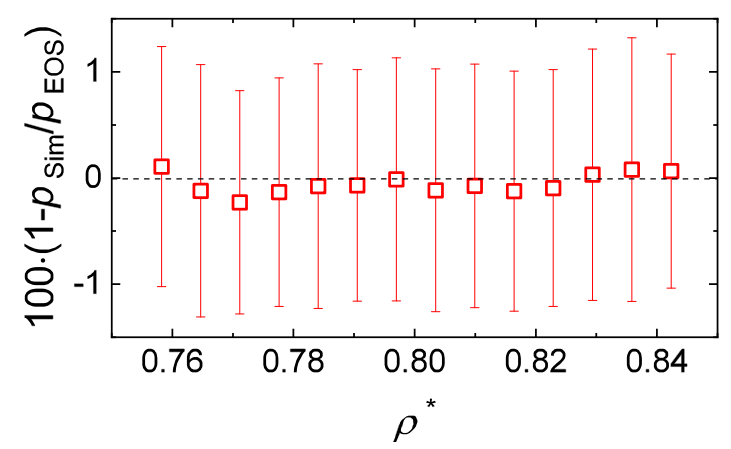

Figure 6.: Comparison of the local pressure with the value predicted from the EOS by Thol et al. [45. The estimated uncertainties are depicted by the error bars. The comparison justifies the hypothesis of local equilibrium for the given scenario.

properties to be in accordance with their equilibrium values, too.

The thermodynamic states and the corresponding transport properties that were determined with the local method are listed in the Supporting Information. Note that the local pressure only slightly fluctuates around the constant value $p^{*}=5.83$.

The local values of $\lambda, \eta$, and $D$ are compared here to correlations of data on transport properties of the LJTS fluid from a comprehensive study on which we will report separately. The deviations are shown in Figure 7. The solid reference line represents the correlations. The dashed lines indicate an uncertainty band of $\pm 10 \%$. The estimated uncertainties of our results are indicated by the error bars. For the scenario investigated here they are mainly below $1 \%$ for $\lambda$, below $5 \%$ for $\eta$, and below $3 \%$ for $D$. The local values are in good agreement with the correlations which represent the equilibrium values. The deviations are below $7.5 \%$ and lie within the scattering of the literature data. The best and most stable results are obtained for $\lambda$ which is due to the large heat flux coming from the large temperature gradient.

\subsection{Shear Rate Study Method}

The temperature gradient is set to $\partial T^{*} / \partial y^{*}=0.03$. The velocity gradient is varied in the range of $\dot{\gamma}^{*}=[0.01 ; 1.0]$. The shear rate dependencies of $\eta, D$, and $p$ are studied at a single state point $\left(T^{*}=0.722, \rho^{*}=0.8442\right)$ which is used as a benchmark in the community [13, 33, 34, 46, 47. The results are shown in Figure 8. They are depicted with the black squares and compared to the data by Heyes [34] (red triangles) and Galea and Attard [13] (blue circles).

The shear rate dependence of $\eta$ found in the present study is in good agreement with the literature data. The well-known shear-thinning effect, i.e. the decrease of $\eta$ with increasing $\dot{\gamma}$, is represented. The deviation from the literature data is mostly below $5 \%$. Our values are slightly higher than the literature data thoroughly.

The shear rate dependence of $D$ found in the present study is compared to results from Heyes [34] in the middle plot of Figure 8, Its general behavior is recursive to the trend of $\eta$. This can be explained by the Stokes-Einstein relation: $D$ behaves inversely proportional to $\eta$. Thus, it increases with increasing $\dot{\gamma}$. The deviations of the present results from those of Heyes [34] are up $17 \%$. This could be related to the high density of the thermodynamic state that was studied here and its proximity to the solidus line.

Contrary to the transport properties, the pressure is strongly affected by the trun- 

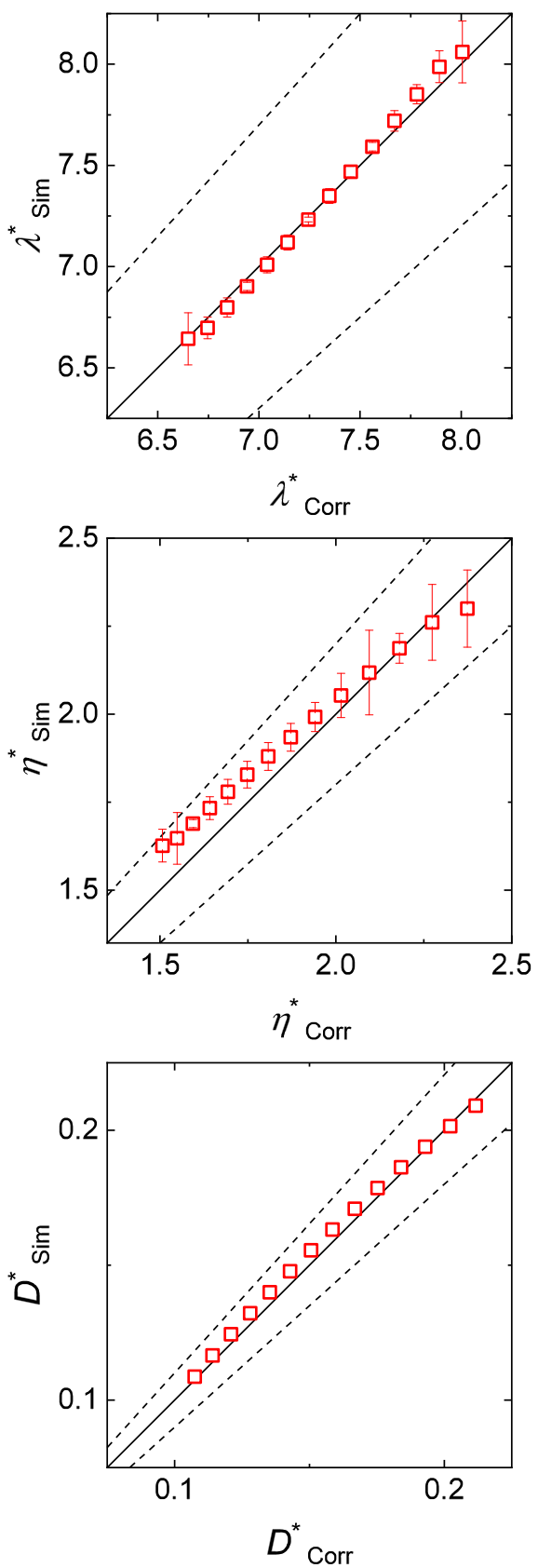

Figure 7.: Deviation plots comparing the transport properties calculated using the local method with correlations from a comprehensive study on which we will report separately. The scenario shown here is $T^{*} \in[1.5 ; 2.5], p^{*}=5.83, \partial T^{*} / \partial y^{*}=0.1$, and $\dot{\gamma}^{*}=0.03$. The solid line represents zero deviation between the simulation results and the correlations. The dashed lines indicate an uncertainty band of $\pm 10 \%$. The estimated uncertainties of our results are depicted by the error bars. 
cation of the LJ potential. Since no LJTS data is available in the literature for $p(\dot{\gamma})$, only our results are shown in the bottom plot of Figure $8, p$ increases with increasing $\dot{\gamma}$. This is in general accordance with the results reported in the literature [33, 34, 46].

\section{Conclusion}

A new non-equilibrium molecular dynamics (NEMD) simulation method for the simultaneous determination of the thermal conductivity $\lambda$ and shear viscosity $\eta$ of fluids, called the two-gradient method, is presented. It is tested using the Lennard-Jones truncated and shifted (LJTS) fluid as example. The influences of the temperature gradient $\partial T / \partial y$ and the shear rate $\partial \boldsymbol{v} / \partial y$ on $\lambda$ and $\eta$ are investigated. The cross-effects are negligible, even for large gradients. Moreover, hardly any influence of $\partial T / \partial y$ on $\lambda$ is observed. However, there is a significant influence of $\partial \boldsymbol{v} / \partial y$ on $\eta$. In addition to the results for $\lambda$ and $\eta$, also results for the self-diffusion coefficient $D$ are determined. The two-gradient method is applied in different ways and the results are compared to the literature. Hereby, it is shown that the two-gradient method is robust, efficient, and yields accurate results. The uncertainties of $\lambda, \eta$, and $D$ are $1 \%, 3 \%$, and $0.5 \%$, respectively, when using the global method. With the local method, they are $1 \%, 5 \%$, and $3 \%$, respectively. The new method is well-suited for studies of liquid and supercritical states as long as the densities are not gas-like. Its accuracy is comparable to that of established methods. The main advantage lies in its efficiency. The new method is, hence, particularly attractive for studies in which for a large number of state points different transport properties of a given fluid shall be determined.

\section{Acknowledgement}

The authors gratefully acknowledge financial support by the DFG within IRTG 2057 Physical Modeling for Virtual Manufacturing Systems and Processes. The simulations were carried out on the elwe at Regional University Computing Center Kaiserslautern (RHRK) under the grant TUKL-TLMV as well as on the SuperMuc at Leibniz Supercomputing Centre (LRZ) Garching under the grant SPARLAMPE (pr48te). The present research was conducted under the auspices of the Boltzmann-Zuse Society for Computational Molecular Engineering (BZS). 


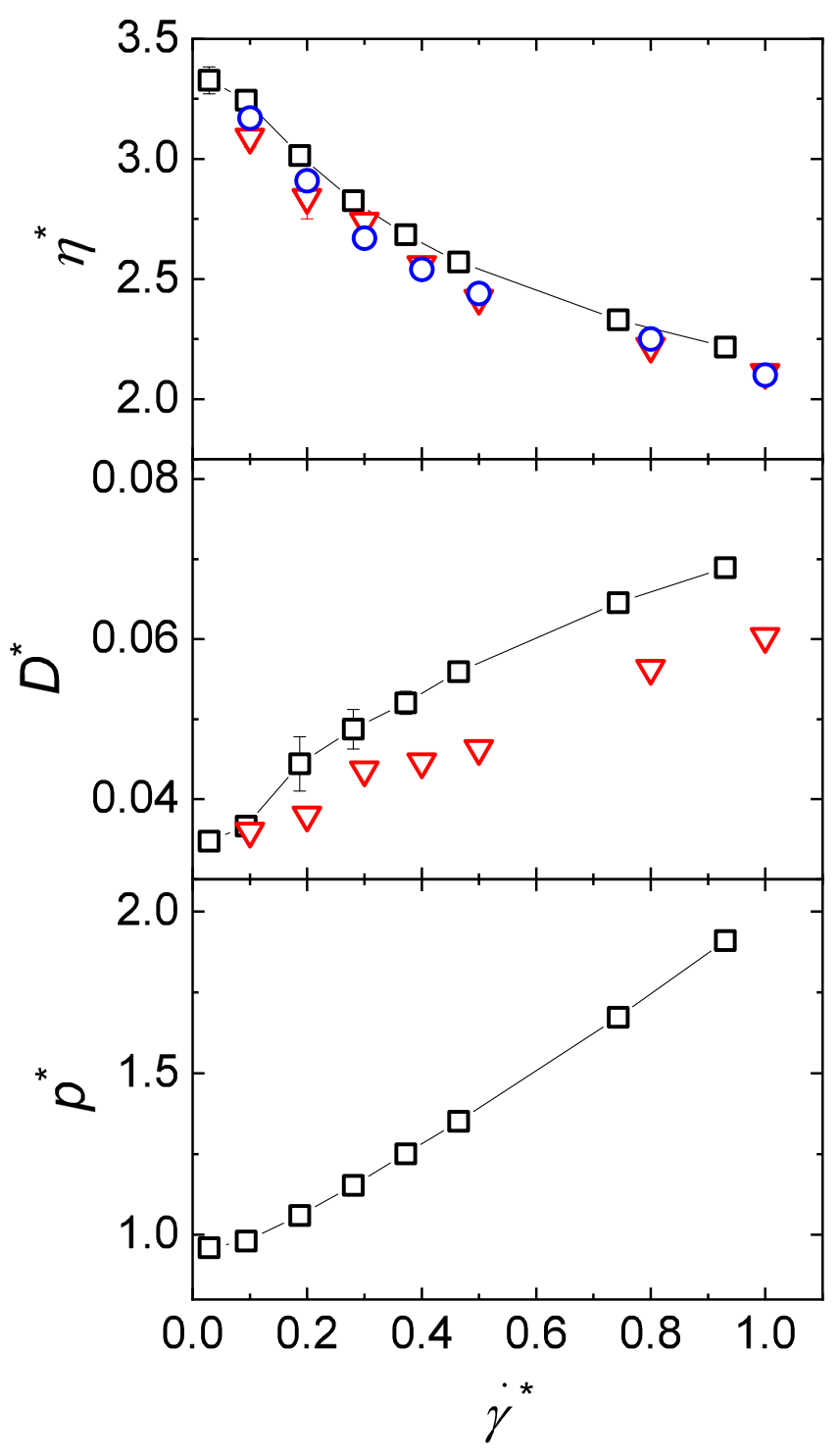

Figure 8.: Shear rate dependence of $\eta$ (top), $D$ (middle), and $p$ (bottom) at a single state $\left(T^{*}=0.722, \rho^{*}=0.8442\right)$. Our values are depicted with black squares. They are compared to the data by Heyes [34] (red triangles) and Galea and Attard [13] (blue circles). The estimated uncertainties of our results are depicted by the error bars. 


\section{Appendix A. Appendices}

\section{Appendix B. Detailed Information}

\section{Directed Velocity.}

In the present work the directed macroscopic flow velocity is averaged over 10,000 time steps in high-resolution bins of size $\left(\Delta x^{*}=1, \Delta y^{*}=1, \Delta z^{*}=L\right)$ each. In dependence on the instantaneous position of a particle $i$ the directed macroscopic flow velocity is assigned to this particle in each time step.

$$
\boldsymbol{v}_{\mathrm{dir}}=\sum_{t=1}^{10,000} \sum_{i=1}^{N_{\mathrm{bin}}} \frac{\boldsymbol{v}_{i}}{10,000 \cdot N_{\mathrm{bin}}}
$$

\section{Error analysis.}

Both the thermal conductivity and the shear viscosity can be written as the ratio of a flux $J$ to its conjugate driving force $\Gamma$; cf. Eqs. (6) \& (7). Thus, the accuracy of the two-gradient method is evaluated by means of the relative approximation error $\Delta \mathrm{TP} / \overline{\mathrm{TP}}$ where $\mathrm{TP}$ stands for the respective transport property; $\mathrm{cf}$. Equation $\mathrm{B} 2$.

$$
\frac{\Delta \mathrm{TP}}{\overline{\mathrm{TP}}} \leq\left(\frac{\Delta J}{\bar{J}}+\frac{\Delta \Gamma}{\bar{\Gamma}}\right)
$$

$\bar{J}$ is the spatial average of the heat flux or shear stress. $\Delta J$ is the empirical variance between the time-averaged simulation data and its corresponding regression fit. $\bar{\Gamma}$ is the spatial average of the derivative of the second-order polynomial fit from the temperature or velocity profile. $\Delta \Gamma$ is the empirical variance from this regression fit. The relative error of the self-diffusion coefficient is estimated by the mean standard deviation of the block-averages. 
Appendix C. Data

The data used in all the figures above is given in the Supporting Information. 


\section{Appendix D. References}

\section{References}

[1] M.S. Green, The Journal of Chemical Physics 20 (8), 1281-1295 (1952).

[2] M.S. Green, The Journal of Chemical Physics 22 (3), 398-413 (1954).

[3] R. Kubo, Journal of the Physical Society of Japan 12 (6), 570-586 (1957).

[4] A. Tenenbaum, Physical Review A 28 (5), 3132-3133 (1983).

[5] D. Evans and P. Cummings, Molecular Physics 72 (4), 893-898 (1991).

[6] B. Hafskjold, T. Ikeshoji and S. Ratkje, Molecular Physics 80 (6), 1389-1412 (1993).

[7] T. Ikeshoji and B. Hafskjold, Molecular Physics 81 (2), 251-261 (1994).

[8] A.W. Lees and S.F. Edwards, Journal of Physics C: Solid State Physics 5 (15), 1921-1928 (1972).

[9] E.M. Gosling, I. McDonald and K. Singer, Molecular Physics 26 (6), 1475-1484 (1973).

[10] W.T. Ashurst and W.G. Hoover, Physical Review A 11 (2), 658-678 (1975).

[11] G. Ciccotti, G. Jacucci and I.R. McDonald, Physical Review A 13 (1), 426-436 (1976).

[12] D.J. Evans and O. Morriss, Computer Physics Reports 1 (6), 297-343 (1984).

[13] T.M. Galea and P. Attard, Physical Review E 66 (4), 041207 (2002).

[14] J.A. Backer, C.P. Lowe, H.C.J. Hoefsloot and P.D. Iedema, The Journal of Chemical Physics 122 (15), 154503 (2005).

[15] F. Müller-Plathe, The Journal of Chemical Physics 106 (14), 6082-6085 (1997).

[16] F. Müller-Plathe, Physical Review E 59 (5), 4894-4898 (1999).

[17] M.P. Allen and D.J. Tildesley, Computer simulation of liquids (Oxford Science Publications, New York, 1989).

[18] P.T. Cummings and D.J. Evans, Industrial \& Engineering Chemistry Research 31 (5), 1237-1252 (1992).

[19] K. Binder, J. Horbach, W. Kob, W. Paul and F. Varnik, Journal of Physics: Condensed Matter 16 (5), S429-S453 (2004).

[20] A. Tenenbaum, G. Ciccotti and R. Gallico, Physical Review A 25 (5), 2778-2787 (1982).

[21] S. de Groot and P. Mazur, Non-Equilibrium Thermodynamics (Dover Publication, New York, 1984).

[22] F. Bresme and J. Armstrong, The Journal of Chemical Physics 140 (1), 016102 (2014).

[23] H. Matsubara, G. Kikugawa, T. Bessho, S. Yamashita and T. Ohara, Fluid Phase Equilibria 421, 1-8 (2016).

[24] F. Bresme, in Joint European Thermodynamics Conference, Vol. 1, pp. 25-28.

[25] D.J. Evans, W.G. Hoover, B.H. Failor, B. Moran and A.J.C. Ladd, Physical Review A 28 (2), 1016-1021 (1983).

[26] J. Irving and J. Kirkwood, Journal of Chemical Physics 18 (06), 817-829 (1950).

[27] M. Bugel and G. Galliéro, Chemical Physics 352 (1-3), 249-257 (2008).

[28] J. Nichele, I. Borges, A.B. Oliveira and L.S. Alves, The Journal of Supercritical Fluids 114, 46-54 (2016).

[29] C. Niethammer, S. Becker, M. Bernreuther, M. Buchholz, W. Eckhardt, A. Heinecke, S. Werth, H.J. Bungartz, C.W. Glass, H. Hasse, J. Vrabec and M. Horsch, Journal of Chemical Theory and Computation 10 (10), 4455-4464 (2014).

[30] G. Karniadakis, A. Beskok and N. Aluru, Microflows and Nanoflows, Vol. Second Edition (Springer-Verlag, New York, 2005).

[31] K.M. Dyer, B.M. Pettitt and G. Stell, The Journal of Chemical Physics 126 (3), 034502 (2007).

[32] G. Rutkai, M. Thol, R. Span and J. Vrabec, Molecular Physics 115 (9-12), 1104-1121 (2017).

[33] D. Heyes, Chemical Physics 109 (1), 47-65 (1986).

[34] D.M. Heyes, Journal of the Chemical Society, Faraday Transactions 282 (9), 1365 (1986).

[35] H.J.M. Hanley and D.J. Evans, The Journal of Chemical Physics 76 (6), 3225-3232 (1982).

[36] K. Takahashi, Entropy 15 (12), 3249-3264 (2013). 
[37] C. Hoheisel, R. Vogelsang and M. Schoen, The Journal of Chemical Physics 87 (12), 7195-7198 (1987).

[38] C. Hoheisel, The Journal of Chemical Physics 86 (4), 2328-2333 (1987).

[39] K. Meier, A. Laesecke and S. Kabelac, The Journal of Chemical Physics 121 (19), 95269535 (2004).

[40] K. Meier, A. Laesecke and S. Kabelac, The Journal of Chemical Physics 121 (8), 36713687 (2004).

[41] V.R. Vasquez, E.A. Macedo and M.S. Zabaloy, International Journal of Thermophysics 25 (6), 1799-1818 (2004).

[42] V. Baidakov, S. Protsenko and Z. Kozlova, Fluid Phase Equilibria 305 (2), 106-113 (2011).

[43] G. Galliéro, C. Boned and A. Baylaucq, Industrial \& Engineering Chemistry Research 44 (17), 6963-6972 (2005).

[44] V.G. Baidakov and S.P. Protsenko, The Journal of Chemical Physics 140 (21), 214506 (2014).

[45] M. Thol, G. Rutkai, R. Span, J. Vrabec and R. Lustig, International Journal of Thermophysics 36 (1), 25-43 (2015).

[46] D. Evans, Physical Review A 23, 1988-1997 (1981).

[47] K.P. Travis, D.J. Searles and D.J. Evans, D., Molecular Physics 95 (2), 195-202 (1998). 